\title{
Micro membrane absorber with deep-permeation nano structure assembled by flowing synthesis
}

\author{
Boya Qiu ${ }^{1}$, Senqing Fan ${ }^{1}$, Yu Chen ${ }^{1}$, Jiaojiao Chen ${ }^{1}$, Yilin Wang ${ }^{1}$, Yinan Wang ${ }^{1}$, Jingyun \\ $\mathrm{Liu}^{1}$, and Zeyi Xiao ${ }^{1}$ \\ ${ }^{1}$ Sichuan University
}

November 16, 2020

\begin{abstract}
A micro membrane adsorber with deep-permeation nano structure (DPNS) has been successfully fabricated by flowing synthesis. The nanoparticles are in situ assembled in membrane pores and immobilized in each membrane pores along the direction of membrane thickness. The nanoparticles with a lower size and thinner size distribution can be achieved owing to the confined space effect of the membrane pores. As a concept-of-proof, the nano ZIF-8 and ZIF-67 are fabricated in porous membrane pores for Methyl orange (MO) and Rhodamine B (RhB) adsorption. The adsorption rate is increased significantly owing to the enhanced contact and mass transfer in the confined space. The adsorption capacity for the RhB is also increased, since the size of the nanoparticles assembled in membrane pores is smaller with more active sites exposed. This micro membrane adsorber with DPNS has good reusability, and can provide a promising prospect for industrial application.
\end{abstract}

\section{Hosted file}

Manuscript .pdf available at https://authorea.com/users/375914/articles/493099-micro-membraneabsorber-with-deep-permeation-nano-structure-assembled-by-flowing-synthesis 